\title{
Testing the correlations between anomalies of statistical indexes of the geoelectric system and earthquakes
}

\author{
Hong-Jia Chen ${ }^{1,2} \cdot$ Chien-Chih Chen ${ }^{1}$
}

Received: 14 December 2015/Accepted: 5 July 2016/Published online: 19 July 2016

(C) The Author(s) 2016. This article is published with open access at Springerlink.com

\begin{abstract}
Geoelectric precursors are considered to be predictors of earthquakes, but this issue is still under debate. The objective of this research is to statistically test the relationship between the geoelectric system and earthquakes. We observed that anomalies of skewness and kurtosis of geoelectric fields may precede large earthquakes. Next, we developed an alarm model of time of increased probability to quantitatively determine their relationship. Performing binary classification and $C 1-F 1$ analysis on both statistical anomalies and earthquake occurrences, the alarm model implies that the statistical correlation between the geoelectric system and earthquakes exists with high confidence. We explained the results by critical transition, which refers to the state of a system becoming slower as it recovers from small perturbations when the system approaches critical points. Hence, generic symptoms, such as autocorrelation, variance, skewness, and kurtosis, can vary appreciably. Early warning signals for critical transitions of the geoelectric system might correspond to impending large earthquakes, in agreement with independent suggestions by other authors that appeared very recently. Consequently, we suggest that the critical transition will take place in the crustal system. Furthermore, we establish a standard procedure to examine the relationship between potential precursor indexes and earthquakes.
\end{abstract}

Keywords Earthquake precursor - Geoelectric field · Time of increased probability · Binary classification · Critical transition

Electronic supplementary material The online version of this article (doi:10.1007/s11069-016-2460-4) contains supplementary material, which is available to authorized users.

Chien-Chih Chen

chencc@earth.ncu.edu.tw

1 Department of Earth Science, National Central University, Jhongli 32001, Taiwan

2 Department of Management, Technology and Economics, ETH Zürich, Zurich, Switzerland 


\section{Introduction}

This work investigates whether anomalies of skewness and kurtosis of geoelectric fields might precede large earthquakes and hence represent an earthquake prediction method. Although the theory of earthquake prediction has not been determined exactly, predicting earthquakes is an emergent task on which a vast volume of literature focuses. From compression experiments of rocks, it is known that electromagnetic radiation (EMR) accompanied by crack growth is a common physical phenomenon (Carpinteri et al. 2010; Fukui et al. 2005; Hadjicontis and Mavromatou 1994; Lacidogna et al. 2011; Mavromatou et al. 2004; Shinbrot et al. 2012; Triantis et al. 2008; Tsutsumi et al. 2003; Frid et al. 2000). Mavromatou et al. (2004) observed that variations in electromagnetic field intensity are coincident with the time derivative of stress, and their amplitudes are almost in peak-topeak correspondence. This correspondence implies that geoelectric fields represent stress variations. Because geoelectric fields are easier to measure than stress, one could install a large set of geoelectric stations to study the relationship between the geoelectric system and earthquakes. Fukui et al. (2005) found, in compression tests, that both rock characteristics and test conditions affected the behavior of EMR generation in rocks under loading. These tests imply that geoelectromagnetic (GEM) signals before earthquakes would vary owing to different geologies. Furthermore, Carpinteri et al. (2010) found that EMR corresponding to each stress drop was detected until peak loads were reached. This evidence enables use of acoustic and electromagnetic emissions as collapse precursors. Although characterizing the connection with GEM changes before, during, and after earthquakes is an open and contested issue in scientific literature, those experimental results support the idea that GEM changes should be related to earthquakes.

In field observations, GEM changes/anomalies have been observed prior to major earthquakes (Astuti et al. 2013; Eftaxias et al. 2003; Huang and Liu 2006; Orihara et al. 2012; Telesca et al. 2004, 2009; Uyeda et al. 2002; Varotsos et al. 1986, 1993, 2002, 2011a, b, c, 2013; Varotsos and Alexopoulos 1984a, b; Varotsos and Lazaridou 1991; Zhao and Qian 1994). In the study of Uyeda et al. (2002), the spectral intensity and the third principal component of geomagnetic fields culminated immediately before nearby earthquakes with $M_{\mathrm{L}} \geq 6$ in the Izu Peninsula. Varotsos et al. (2002) found that the seismic electric signals (SESs) preceding the 13 May 1995 Kozani-Grevena earthquake exhibited scaling laws. The scaling laws imply that long-range correlations exist in the crustal system. Their laboratory measurements reinforce the suggestion that emission of SES activity could be discussed in the frame of the theory of dynamic phase transitions. Eftaxias et al. (2003) observed that electromagnetic anomalies can cover a wide range of frequencies, and the power spectral densities of those SESs show that the power densities in low-frequency bands increase before major earthquakes and that powerlaw exponents increase from 1 to 2 . Their results imply an underlying critical transition in the underground and suggest an underlying structural instability that belongs to the final stage of the earthquake preparation process.

Nevertheless, most previous works only presented cases of observed earthquakes with precursors (true positives), but not false-positive or false-negative cases. Therefore, we do not know whether the defined precursory indexes are correlated with earthquakes or the prediction method has good performance. As sufficient data on both geoelectrics and earthquakes are available for Taiwan, one might solve the uncertainty regarding whether an index is precursory for earthquakes. A geoelectric monitoring system (GEMS) was installed on Taiwan Island from the beginning of 2012. The chief aim of the GEMS network is to 
understand the possibility of the production of enormous electric dipoles and potential changes near seismogenic zones. On the other hand, there are approximately 20 earthquakes per year with $M_{\mathrm{L}} \geq 5$ in Taiwan. Hence, we can collect sufficient datasets to analyze the relationship between the geoelectric system and earthquakes. The purpose of this study is to statistically test the relationship between the geoelectric system and earthquakes based on data from the dense geoelectric stations and abundant earthquakes in Taiwan. Referring to Huang's thoughts (2011), we attempted to build an alarm model considering a physics-based approach. In this paper, we have referred to the concept of time of increased probability (TIP) from Keilis-Borok and Kossobokov (1990), applied the TIP concept on the GEMS datasets, and then developed a GEMSTIP algorithm which can determine the relevant precursor indexes and estimate the alarm model performance. Based on the analysis of the GEMSTIP algorithm on the geoelectric fields and the earthquake catalog, we suggest that there are statistical correlations between the geoelectric system and earthquakes.

\section{Data}

In this work, the datasets consisted of (1) geoelectric fields from the GEMS and (2) the earthquake catalog from the Central Weather Bureau (CWB) of Taiwan.

\subsection{Geoelectric fields}

At the beginning of 2012, 20 stations were deployed one after another to monitor geoelectric fields continuously in the Taiwan region; the geoelectric network is named GEMS. The GEMS stations were extensively and uniformly installed on Taiwan Island (Fig. 1).

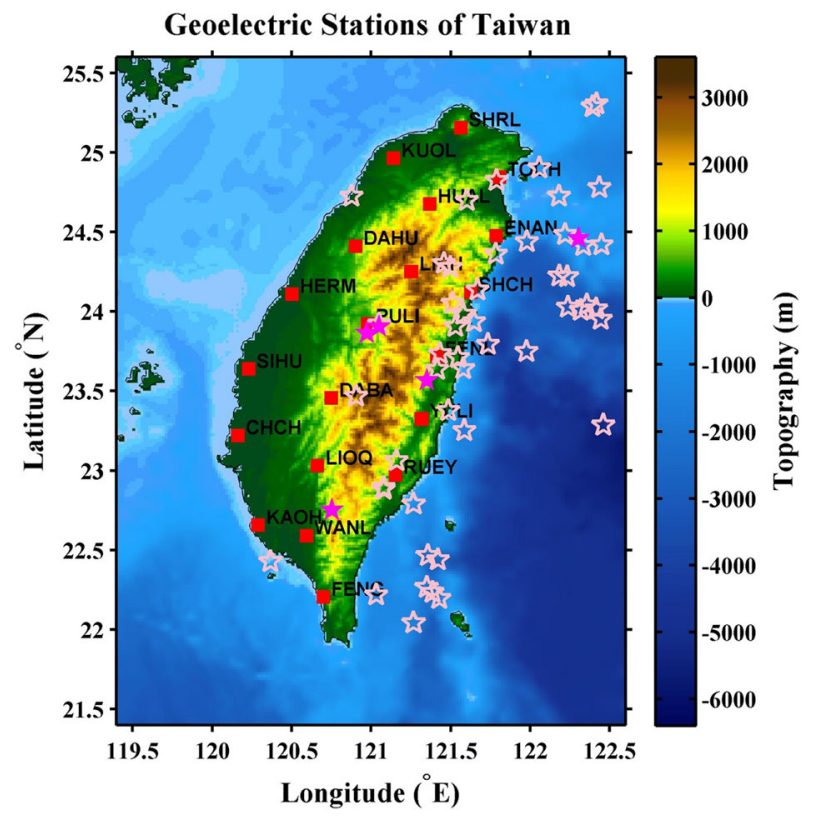

Fig. 1 Spatial distribution of geoelectric stations and major earthquakes in Taiwan. Red squares are geoelectric stations, magenta stars are earthquakes with $M_{\mathrm{L}} \geq 6$, and open pink stars are earthquakes with $M_{\mathrm{L}} \in[5,6)$. The earthquake catalog is from $2012 / 1 / 1$ to $2014 / 12 / 31$ 
The spacing between neighboring stations is approximately $50 \mathrm{~km}$. Geoelectric fields registered by GEMS are equivalent to self-potentials, which are naturally occurring electric potential differences in the Earth. Self-potentials are passively measured at the ground surface or in boreholes by means of a nonpolarizable electrode relative to a fixed reference one. There are two long dipoles connected with telephone cables to transport electric signals at each GEMS station. The two horizontally deployed dipoles stretch for approximately 1-4 km and share a reference electrode. Because of site limitations, the azimuths of the two dipoles might be tilted by approximately $10-40^{\circ}$ from exact north and exact east, respectively. We thus preprocessed the tilted geoelectric fields to north-south (NS) and east-west (EW) components. The GEMS database is available and can be requested from Prof. Chien-Chih Chen (chencc@earth.ncu.edu.tw) in the Department of Earth Science of National Central University, Taiwan. In this work, we used the data of geoelectric fields from the starting time of each GEMS station until 2014/12/31; the geoelectric fields had sampling frequency of $1 \mathrm{~Hz}$ and units of $\mathrm{mV} / \mathrm{km}$.

\subsection{Earthquake catalog}

An earthquake catalog for the Taiwan region is routinely released and maintained by the CWB of Taiwan (http://www.cwb.gov.tw/V7e/earthquake/). The system of the CWB seismic network has been upgraded since 2010: the seismic signals are now digitized at 24 instead of 12 bits, and the number of stations has increased, with more than 100 now widely installed in the Taiwan region. The types of seismic instruments include shortperiod seismographs, accelerometers, and broadband instruments. The source parameters of an earthquake can, therefore, be estimated more accurately. Details of the seismic network in Taiwan are presented in the papers by Chang et al. (2012) and Hsiao et al. (2011). In this study, we used the earthquakes with $M_{\mathrm{L}} \geq 5$ that occurred from 2012/01/01 to $2014 / 12 / 31$ in the area of $119.5-122.5 \mathrm{E}, 21.5-25.5 \mathrm{~N}$ of Taiwan. Figure 1 illustrates the spatial distribution of epicenters of the selected earthquakes. We found that the large earthquakes mainly occurred in eastern Taiwan, while only two occurred near the coast of western Taiwan (near DAHU and $\mathrm{KAOH}$, respectively). In total, 55 large earthquakes were chosen. The number of earthquakes with $M_{\mathrm{L}} \in[5,6)$ was 50 , and the number of those with $M_{\mathrm{L}} \geq 6$ was 5 .

\section{Methods}

In this work, we used the following concepts and analysis methods: calculations of skewness and kurtosis, a definition of anomaly, the time of increased probability (TIP), coarse-graining of earthquake occurrences, binary classification, $C 1-F 1$ analysis, and a significance test.

\subsection{Calculations of skewness and kurtosis}

Based on the concept of early warning signals for critical transitions (Scheffer et al. 2009), we understood that statistical indexes, such as autocorrelation, variance, skewness, and kurtosis, would change anomalously preceding catastrophic events. These indexes might identify periods of critical transitions. Here, we chose skewness and kurtosis to test the relationship between the geoelectric system and earthquakes. 
In statistics, skewness (sk) is the standardized third moment around the mean of a distribution, defined as follows:

$$
\mathrm{sk}=\frac{E(x-\mu)^{3}}{\sigma^{3}},
$$

where $x$ represents the observed data points, $\mu$ is the mean of $x, \sigma$ is the standard deviation of $x$, and the operator $E(\cdot)$ represents the expected value. Skewness is a measure of the asymmetry of the data around the mean. The skewness of a normal distribution (or any perfectly symmetric distribution) is 0 . If the skewness is negative, the data are spread out more to the left of the mean than to the right. If the skewness is positive, the data are more to the right. Kurtosis $(\mathrm{ku})$ is the standardized fourth moment around the mean of a distribution, defined as follows:

$$
\mathrm{ku}=\frac{E(x-\mu)^{4}}{\sigma^{4}},
$$

where the meanings of the symbols are the same as for Eq. (1). Kurtosis is a measure of how sharp and narrow a distribution is. The kurtosis of a normal distribution is 3 . Distributions that are more outlier-prone than the normal distribution have kurtosis values greater than 3 (leptokurtic); distributions that are less outlier-prone have kurtosis values less than 3 (platykurtic).

In this work, we calculated these two indexes for the time series of geoelectric fields every day, and we obtained the daily variations of skewness and kurtosis. In reality, the ideal, normal distribution may seldom appear. As a result, one needs to apply thresholds to identify practical distributions.

\subsection{Definition of anomaly}

An anomaly is a deviation from the normal type, rule, arrangement, or form. Operationally, we assumed a lower and an upper threshold ( $L_{\mathrm{thr}}$ and $\left.U_{\mathrm{thr}}\right)$ to distinguish anomaly from normal. These were defined as follows:

$$
\begin{gathered}
\mathrm{IQR}=Q_{3}-Q_{1}, \\
L_{\mathrm{thr}}=\text { Median }-3 \mathrm{IQR}, \\
U_{\mathrm{thr}}=\text { Median }+3 \mathrm{IQR} .
\end{gathered}
$$

The variables $Q_{1}, Q_{3}$, and IQR denote the first quartile, third quartile, and interquartile range, respectively. We generally determined values between $L_{\mathrm{thr}}$ and $U_{\text {thr }}$ to be normal, and values outside of this range to be anomalous. We thereby estimated the thresholds for the skewness and kurtosis series for each station.

\subsection{Alarm model: time of increased probability of GEMS}

The term "time of increased probability" (TIP) comes from the works of Keilis-Borok and Kossobokov (1990). They defined TIP by means of premonitory intermediate-term seismic activation in the lower-magnitude range, and they explored the possibility of the occurrence of a strong earthquake. They showed that seismic activation is the relevant preparation process for an impending catastrophic earthquake by means of TIP analysis. 
In this work, according to the anomalies of skewness and kurtosis of geoelectric fields, we developed our TIP model, GEMSTIP, with parameters $\operatorname{Rad}(\mathrm{km})$, Dep $(\mathrm{km}), N, t 1$ (days), $t 2$ (days), and $t 3$ (days). Earthquakes were selected based on the parameters Rad and Dep; For example, $\operatorname{Rad}=55$ and Dep $=30$ at PULI means that earthquakes within a radius of $55 \mathrm{~km}$ from the center of PULI and above depth of $30 \mathrm{~km}$ were selected. The parameter $N$ is the threshold number for the anomalous indexes, determining that a day is labeled as anomalous if the anomaly index number (AIN) $\geq N$. The maximum AIN is 4 because there are two statistical indexes per component and two components per station. The parameters $t 1, t 2$, and $t 3$ are time windows with units of days. The definition of TIP is as follows: First, a day is defined as anomalous if AIN $\geq N$. Then, if the number of anomalous days within the window $t 2$ is greater than $t 1$ days, the future window $t 3$ is defined as time of increased probability (TIP index). TIP indexes are $0-1$ binary series, in which 0 means no TIP and 1 means TIP.

In this work, $\operatorname{Rad} \in[1,30: 5: 55,60: 10: 100,999](\mathrm{km}), \operatorname{Dep} \in[1,30: 30: 300,999](\mathrm{km})$, $N \in[1,2,3,4], t 1 \in[1: 1:(2 / 3) \times t 2]$ (days), $t 2 \in[1,5: 5: 30,40: 10: 90]$ (days), and $t 3 \in[1,5: 5: 30,40: 10: 90]$ (days), where the data format is [start:increment:end]. Therefore, the total number of GEMSTIP models used was 2,717,520.

\subsection{Coarse-graining of earthquake occurrences}

Coarse-graining is similar to adjusting the objective working distance in the observation of cells with a microscope. If the working distance is too close or too far, one cannot discern the structures of cells well, and one can observe the structures clearly only with the appropriate working distance. The theory of granularity is used in many fields, including physics, molecular dynamics, computing, etc. In seismology, the concept of coarsegraining has also been applied to investigate fault dynamics (Abe and Suzuki 2009; Klein et al. 1997; Tiampo et al. 2002).

In this study, it is inequitable that the time resolution of an earthquake is one time point whereas the resolution of the predictive time of a GEMSTIP model is $t 3$ (days). Therefore, we developed a coarse-grained method to expand earthquake occurrence times. In the coarse-graining procedure, earthquake occurrence times (EQ indexes) are extended plus and minus $t 3 / 2$ days (CGEQ indexes); hence, the CGEQ indexes are determined by the parameters Rad, Dep, and $t 3$. EQ and CGEQ indexes are $0-1$ binary series, in which 0 means no EQ and 1 means EQ. On the other hand, the multifractal stress activation model (Ouillon et al. 2009; Ouillon and Sornette 2005; Sornette and Ouillon 2005; Tsai et al. 2012) predicts that an earthquake will probably occur in a time window if the crustal state (stress) reaches a threshold. The time window is related to the nucleation of an event whose dynamical occurrence is unknown, so it may vary from event to event due to disorder and differences in initial conditions; therefore, we treated it using an unknown, finite-size window (personal communication with Ouillon 2016). Hence, coarse-graining the earthquake occurrence time and predicting earthquake-prone periods using the GEMSTIP algorithm is a plausible approach.

\subsection{Binary classification and $C 1-F 1$ analysis}

Binary classification is heavily used in many areas, especially medicine and machine learning. This classification method forms a 2-by-2 contingency table, including the number of true positives (TP), false positives (FP), false negatives (FN), and true negatives (TN), which can determine whether the classifying model behaves well. In summary, the 
greater the proportions of TN and TP, the better the performance of the classifying model. In medicine, for example, a diagnostic or medical test can reveal the incidence of cancer with a positive test or the incidence of no cancer with a negative test. The resulting portions can elucidate whether the testing method is reliable to inform patients about their health condition.

To quantify the performance of a GEMSTIP model, we introduce two indexes that can estimate the proportions of TN and TP in the four classes. The two indexes are $C 1$ and $F 1$, defined as follows:

$$
\begin{aligned}
& C 1=\frac{2 \mathrm{TN}}{2 \mathrm{TN}+\mathrm{FP}+\mathrm{FN}}, \\
& F 1=\frac{2 \mathrm{TP}}{2 \mathrm{TP}+\mathrm{FP}+\mathrm{FN}} .
\end{aligned}
$$

The values of both $C 1$ and $F 1$ range from 0 to 1 . We also define $(C 1, F 1)=(0,1)$ when $\mathrm{TP}=1, \mathrm{FP}=0, \mathrm{FN}=0$, and $\mathrm{TN}=0$, and we define $(C 1, F 1)=(1,0)$ when $\mathrm{TP}=0$, $\mathrm{FP}=0, \mathrm{FN}=0$, and $\mathrm{TN}=1$. This assignment can avoid the occurrence of not-a-number. The value $C 1=0$ means that no true negatives exist, whereas $C 1=1$ means all true negatives. The value $F 1$ has similar descriptions.

Based on $C 1$ and $F 1$, one can determine the ability to identify TN and TP in the four classes. A good performance model must have large values of both $C 1$ and $F 1$. Hence, we define the model performance $R$ as follows:

$$
R=\sqrt{C 1^{2}+F 1^{2}} .
$$

If $C 1=1$ and $F 1=1$, then $R=R_{\text {perf }}=\sqrt{ } 2$; the value $R_{\text {perf }}$ means that the predictive model has perfect performance and the observed and predicted datasets match each other perfectly. The value $R$ can be considered as the matching coefficient. A higher $R$ value suggests that there is a potential model connecting the geoelectric system and earthquakes.

In this work, after obtaining the CGEQ and TIP indexes, we compared the two 0-1 binary series and formed a 2-by-2 contingency table. TP means successful forecast $(\mathrm{TIP}=$ yes, CGEQ = yes), FP means false alarm $(\mathrm{TIP}=$ yes, CGEQ $=$ no), FN means missing alarm (TIP $=$ no, CGEQ $=$ yes $)$, and TN means successful forecast of nonoccurrence $(\mathrm{TIP}=$ no, CGEQ $=$ no). We then conducted $C 1-F 1$ analysis on the TP, FP, FN, and TN of the GEMSTIP models with different parameters [Rad, Dep, $N, t 1, t 2, t 3]$. In practice, the best GEMSTIP model was defined as the one having maximal performance $R$ in the models without end parameters. We avoided models with end parameters, called end models, which cannot converge to the upper or lower boundary of any input parameter. Moreover, we neglected models without CGEQ indexes or TIP indexes, meaning there is no earthquake or no anomaly to constrain the GEMSTIP model. This maximal $R$ is called $R_{\text {best }}$, and the parameters of the best GEMSTIP model are $\left[\operatorname{Rad}_{\text {best }}, D_{\text {epest }}, N_{\text {best }}, t 1_{\text {best }}\right.$, $\left.t 2_{\text {best }}, t 3_{\text {best }}\right]$.

\subsection{Significance test}

After obtaining the parameters $\left[\operatorname{Rad}_{\text {best }}\right.$, Dep best $\left., N_{\text {best }}, t 1_{\text {best }}, t 2_{\text {best }}, t 3_{\text {best }}\right]$ for all stations, we tested the significance of this best parameter set. First, we computed the interevent times of anomalous indexes of skewness and kurtosis per component. The interevent time is the time elapsed between two successive anomalous indexes. Second, we randomly 

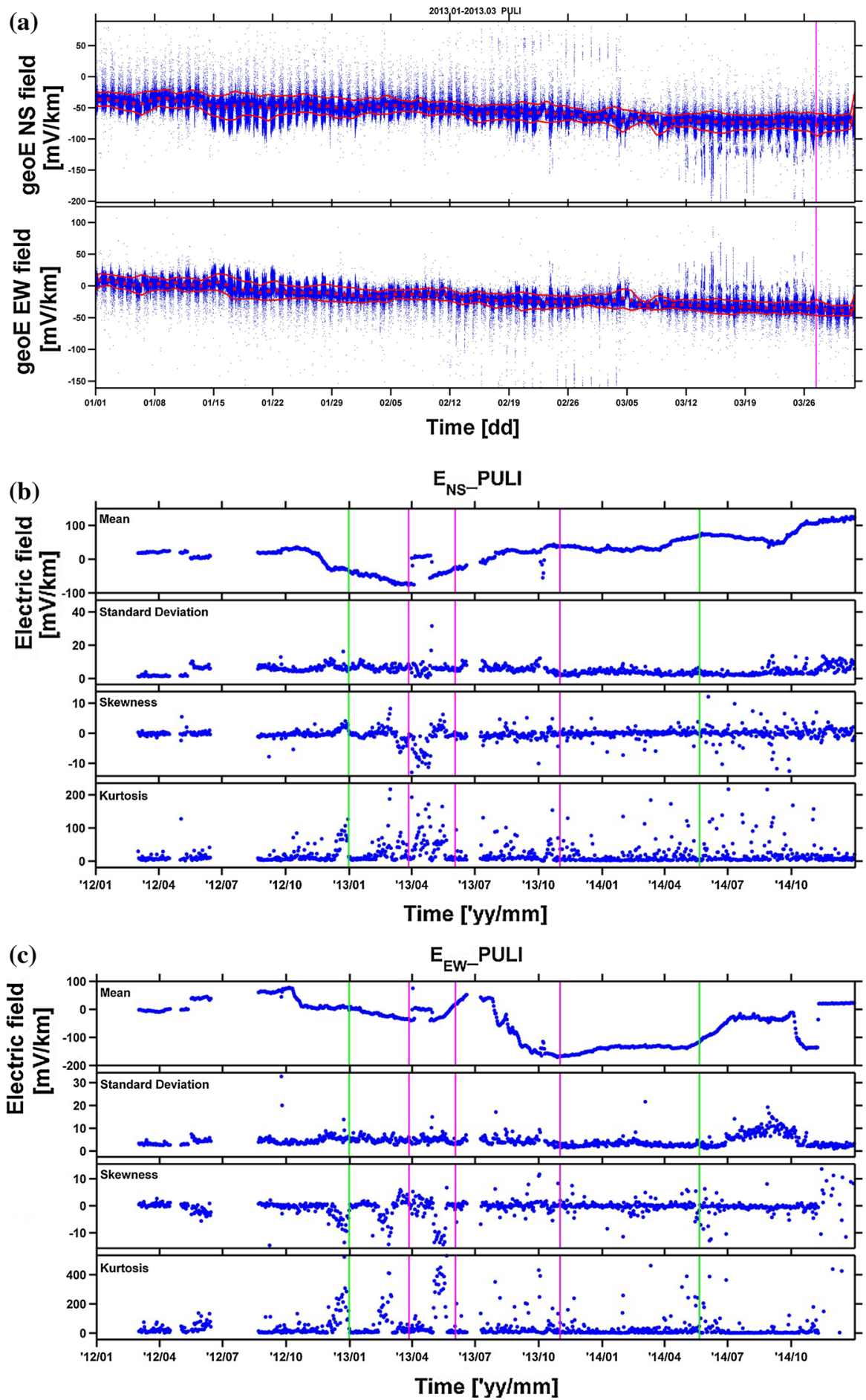
Fig. 2 a NS and EW geoelectric fields at PULI with 1-Hz sampling rate from 2013/1/1 to 2013/3/31. Red crosses are daily means, and red lines show one standard deviation. PULI's statistical indexes of (b) NS component and (c) EW component from 2012/3/1 (starting time of PULI) to 2014/12/31. In (b) and (c), the first row is the mean, the second is the standard deviation, the third is the skewness, and the final is the kurtosis. Vertical lines denote larger earthquakes within a radius of $55 \mathrm{~km}$ around PULI. Magenta verticals are earthquakes with $M_{\mathrm{L}} \geq 6$, and green verticals are those with $M_{\mathrm{L}} \in[5,6)$

shuffled the interevent times, accumulated them into the original time coordinate, and generated surrogate series of skewness and kurtosis per component. Finally, we optimized the GEMSTIP model using the surrogate datasets and the earthquake catalog. These surrogate datasets are theoretically independent of the earthquake occurrence times. We repeated this optimization 200 times, obtaining 200 parameter sets for the best GEMSTIP model from the surrogate datasets for each station.

We then developed a diagnosis to test whether an $N$-dimensional point $(X)$ is included in the $N$-dimensional dataset of $M$ points $\left(Y_{i}\right)$. The null hypothesis $H^{0}$ is that $X$ is included in $Y_{i}$. We then calculated the following Euclidean distance $D_{i}$ between $X$ and $Y_{i}$ and $D_{i j}^{0}$ among $Y_{i}$, respectively:

$$
\begin{gathered}
D_{i}=\left\|X-Y_{i}\right\| \text { for } i=1, \ldots, M \\
D_{i j}^{0}=\left\|Y_{i}-Y_{j}\right\| \text { for } i, j=1, \ldots, M \text { and } i \neq j .
\end{gathered}
$$

We transformed the original $H^{0}$ to become that $D_{i}$ and $D_{i j}^{0}$ are from the same continuous distribution. We used a Kolmogorov-Smirnov (KS) test on $D_{i}$ and $D_{i j}^{0}$, which examines whether two distributions are from the same population (Massey 1951; Miller 1956).

$$
\mathrm{KS}_{j}=\mathrm{KS} \text { test }\left(D_{i}, D_{i j}^{0}, \alpha\right)
$$

The value $\alpha$ is the significance level; here, $\alpha=0.05$. The result $\mathrm{KS}_{j}$ is 1 if the test rejects the null hypothesis at $5 \%$ significance level, and 0 otherwise. Finally, we define the index $H$ as follows:

$$
H=\frac{\sum_{j=1}^{M} \mathrm{KS}_{j}}{M}>0.95 .
$$

If the ratio of the number of rejections to the total number is greater than 0.95 , then $H=1$ and we reject that $X$ is included in $Y_{i}$; otherwise it is 0 . In this paper, $X$ is the best parameters of the real case for each station, $Y_{i}$ is those of the random cases of each station, the dimension is $6\left[\left(\operatorname{Rad}_{\text {best }}, \mathrm{Dep}_{\text {best }}, N_{\text {best }}, t 1_{\text {best }}, t 2_{\text {best }}, t 3_{\text {best }}\right)\right]$, and $M$ is 200 .

\section{Results}

Two devastating earthquakes with $M_{\mathrm{L}}>6$ occurred near PULI, on 2013/03/27 and 2013/06/02. Figure 2a shows the 1-Hz geoelectric fields of PULI's NS and EW components from $2013 / 1 / 1$ to $2013 / 3 / 31$. We observe that the data are spread more to the lower part in the NS component and more to the upper part in the EW component before the 2013/03/27 earthquake. Further analyzing the geoelectric fields at PULI, there are 
anomalous statistics of the geoelectric fields before major earthquakes. Figure $2 \mathrm{~b}$ and $\mathrm{c}$ show the daily variations of the mean, standard deviation, skewness, and kurtosis of PULI's NS and EW components, respectively (hereinafter denoted $E_{\text {NS_PULI }}$ and $\left.E_{\mathrm{EW} \_} \mathrm{PULI}\right)$; For example, the daily mean of $E_{\mathrm{NS} \_}$PULI ranges roughly from -70 to $120 \mathrm{mV} / \mathrm{km}$ and exhibits a bowl shape from 2012/11/08 to 2013/10/01 when the two earthquakes occurred. This pattern is strikingly reminiscent of the so-called gradual variation of the electric field of the Earth identified in Greece (see also p. 8 of the book The Physics of Seismic Electric Signals by Varotsos 2005; Varotsos and Alexopoulos 1984b). The background of the daily standard deviation is below approximately $10 \mathrm{mV} / \mathrm{km}$, and only few outliers appear before the two earthquakes. However, meanwhile, the values of skewness deviate away from the normal range approximately between -2 and 2 . Conventionally, a distribution is thought of as symmetric when its skewness is in this range. Additionally, the kurtosis values are beyond the threshold of approximately 50. In summary, the distributions become narrower and sharper than normal and show longer tails to the right or left before large earthquakes. Besides, there are time lags between earthquake occurrences and the clusters of anomalous skewness and anomalous kurtosis; For example, the time lag between the peak of the cluster of negative skewness (2013/03/15) and the $\left\{2013 / 03 / 27,121.05 \mathrm{E}, 23.90 \mathrm{~N}, M_{\mathrm{L}} 6.24\right\}$ earthquake was 12 days, whereas that between the peak of the cluster of positive skewness $(2013 / 05 / 16)$ and the $\{2013 / 06 / 02,120.97 \mathrm{E}$, $\left.23.86 \mathrm{~N}, M_{\mathrm{L}} 6.48\right\}$ earthquake was 17 days. Similar observations also appear in $E_{\mathrm{EW}}$ PULI. These time lags are comparable to those observed between SESs and earthquakes in Greece (Varotsos et al. 1993, 2009) and Japan (Varotsos et al. 2013). If we select more earthquakes with $M_{\mathrm{L}} \geq 5$ and within a radius of $55 \mathrm{~km}$ around PULI, there are two more earthquakes, i.e., $\left\{2012 / 12 / 31,120.91 \mathrm{E}, 23.47 \mathrm{~N}, M_{\mathrm{L}} 5.28\right\}$ and $\{2013 / 10 / 31,121.35 \mathrm{E}$, $\left.23.57 \mathrm{~N}, M_{\mathrm{L}} 6.42\right\}$, with preceding abnormal clusters of skewness and kurtosis.

These coincidences between larger earthquakes and anomalies of skewness and kurtosis of PULI's geoelectric fields prompted this study to analyze, comprehensively and systematically, the correlation between major earthquake occurrences and the skewness and kurtosis of geoelectric fields for all stations. Figures S1, S2, S3, and S4 show the skewness of both the NS and EW components and the kurtosis of both the NS and EW components,

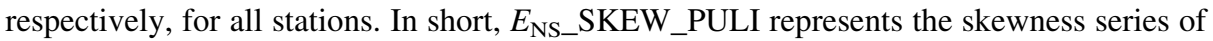
geoelectric fields of PULI's NS component, and $E_{\mathrm{EW}}$ KURT_PULI represents the kurtosis series of geoelectric fields of PULI's EW component, and so forth. First, we observed the skewness of the geoelectric fields in the NS components for all stations (Fig. S2). In

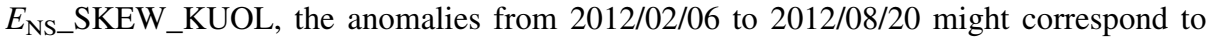
the $\left\{2012 / 08 / 31,120.88 \mathrm{E}, 24.72 \mathrm{~N}, M_{\mathrm{L}} 5.04\right\}$ earthquake. Additionally, in $E_{\mathrm{NS}}$ SKEW_DABA, the anomalies from 2013/02/25 to 2013/05/17 correspond to the $\{2013 / 06 /$ $\left.02,120.97 \mathrm{E}, 23.86 \mathrm{~N}, M_{\mathrm{L}} 6.48\right\}$ earthquake. However, in $E_{\mathrm{NS}}$ SKEW_SIHU, the anomalies from $2014 / 04 / 02$ to $2014 / 09 / 04$ do not correspond to earthquakes with $M_{\mathrm{L}} \geq 5$. Also, in

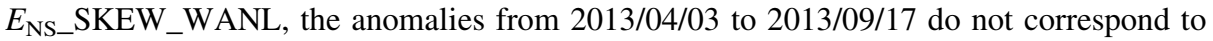
earthquakes with $M_{\mathrm{L}} \geq 5$. For most other periods, there are no anomalies and no events. Similar observations appear in Figs. S3, S4, and S5. Major questions can be raised after visual observation of these figures. First, sometimes there are many anomalies before earthquakes, but sometimes there are few. One may ask: How many anomalies are needed to identify impending earthquakes? And secondly: What percentage of anomalies have no corresponding earthquakes? And what percentage of earthquakes have no corresponding anomalies? These questions seem to be solved by binary classification between earthquake occurrences and the anomalies of skewness and kurtosis of the geoelectric fields. 
We established a GEMSTIP model with parameters [Rad, Dep, $N, t 1, t 2, t 3$ ] to compare statistical anomalies with earthquake occurrences. Figure 3 shows the daily series of PULI's anomalous index numbers, TIP and CGEQ indexes, and its contingency table for the specific model with [Rad, Dep, $N, t 1, t 2, t 3]=[55,30,2,5,15,30]$. The earthquakes were selected using the condition $\operatorname{Rad}=55(\mathrm{~km})$ and Dep $=30(\mathrm{~km})$ around PULI. There is more than one statistical index at PULI showing anomalies prior to major earthquakes in the upper panel of Fig. 3. The duration of anomalous index numbers is several days before those earthquakes, which elucidates that those anomalies would not come from temporal cultural noises before large earthquakes. Based on the GEMSTIP model, we obtained the TIP index (blue line) and EQ index (red star) shown in the lower panel of Fig. 3. We then coarse-grained the time of the EQ index to obtain the CGEQ index (green line). Applying binary classification on the TIP and CGEQ indexes of this model resulted in a 2-by-2 contingency table with $\mathrm{TP}=100, \mathrm{FP}=225, \mathrm{FN}=55$, and $\mathrm{TN}=640$ (inset of lower panel). Coarse-graining of earthquake occurrences is more discernible in the binary classification, as shown in Fig. 4a. If earthquake occurrence is not coarse-grained, the $F 1$ values of all models will be suppressed in the lower $F 1$-axis. If coarse-graining is applied, the $F 1$ values are extended in all $F 1$-axes, revealing the detailed structure in the $C 1-F 1$ scatter plot.

We analyzed 2,717,520 GEMSTIP models for each station, and estimated their performance. Each model generates the TIP and CGEQ indexes and corresponds to one $(C 1$, $F 1)$ pair in the $C 1-F 1$ scatter plot (Fig. $4 \mathrm{~b})$. The ideal GEMSTIP model has the pair $(C 1$, $F 1)=(1,1)$ with matching coefficient $R_{\text {perf }}=\sqrt{ } 2$, and we draw the reference line $\left(R=1 \fallingdotseq R_{\text {perf }} \times 0.7\right)$ for comparison. Note that we ignored models with end parameters (shown as grey dots), for which the performance might not converge. We then found the

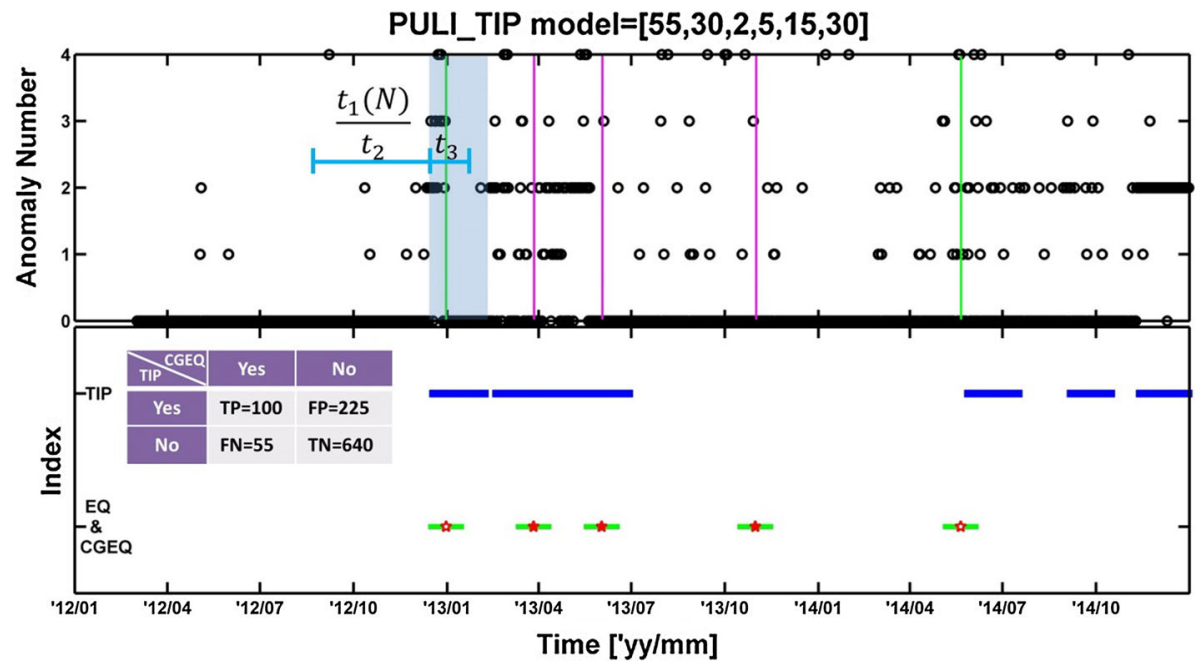

Fig. 3 Schematic diagram of a GEMSTIP model. The upper panel shows the time series of anomaly index number (AIN) at PULI, denoted by black circles. Vertical lines are earthquakes with $M_{\mathrm{L}} \geq 5$, selected using the parameters $\mathrm{Rad}=55 \mathrm{~km}$ and Dep $=30 \mathrm{~km}$ at PULI. Magenta verticals are earthquakes with $M_{\mathrm{L}} \geq 6$, and green verticals are those with $M_{\mathrm{L}} \in[5,6)$. The maximum AIN each day is 4 . The lower panel shows the time series of the TIP and CGEQ indexes from the GEMSTIP model [55, 30, 2, 5, 15, 30]. The definition of TIP is as follows: if there are $t 1$ with $\mathrm{AIN} \geq N$ within a window of $t 2$, then the future $t 3$ is an earthquakeprone period. A contingency table based on the model is constructed in the inset of the lower panel 

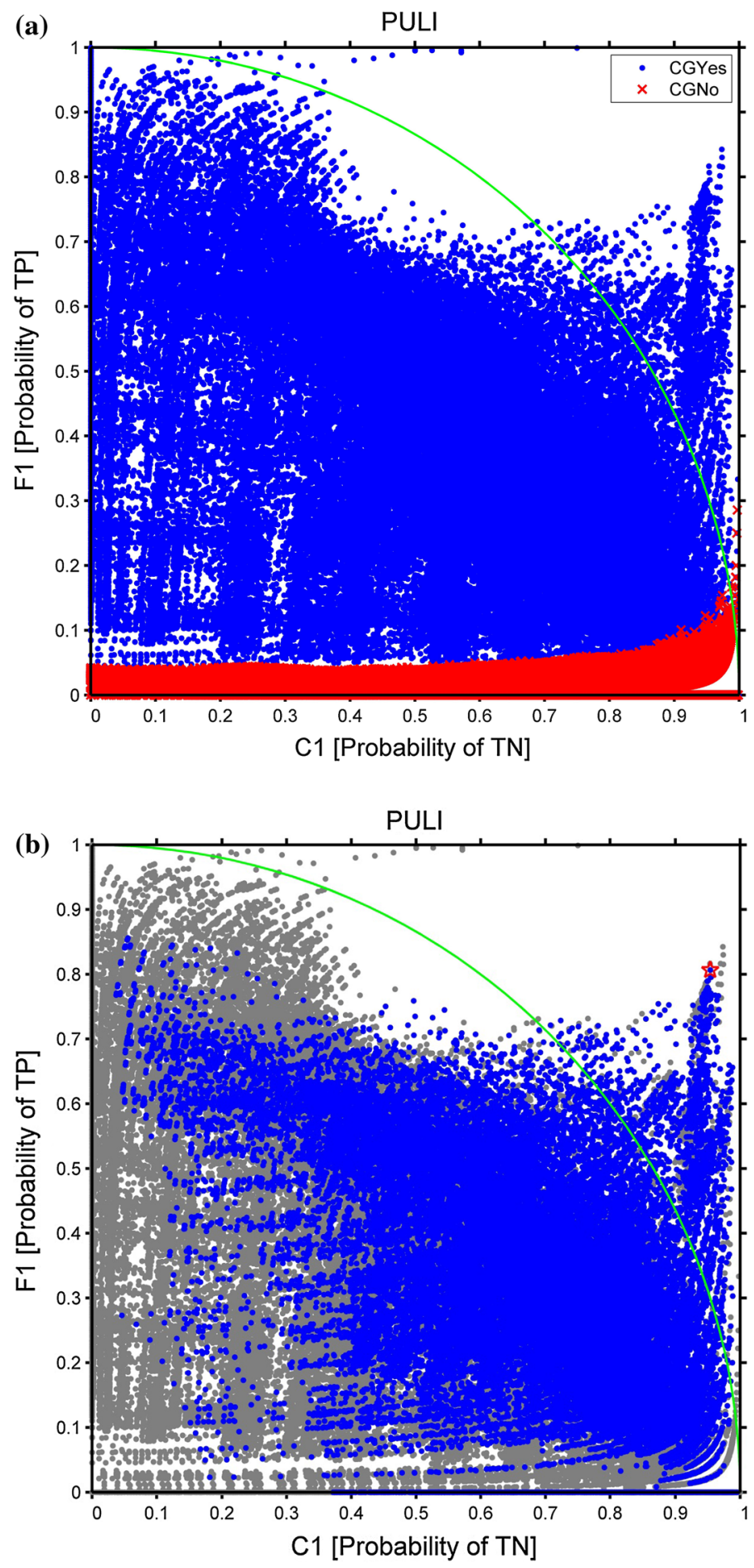
Fig. 4 a $C 1-F 1$ scatter plot at PULI for coarse-graining case (blue dots) and non-coarse-graining case (red crosses). b $C 1-F 1$ scatter plot at PULI. Blue dots are results of TIP models without end parameters, while grey dots indicate end models. The red star is the best result of the TIP models. Generally, the greater the distance from the origin, the better the performance of the TIP model. The green line is for reference, indicating $R=1=R_{\text {perf }} \times 0.7$

best GEMSTIP model among the non-end models. At PULI, the best GEMSTIP model (marked by a red star) has $(C 1, F 1)=(0.95,0.80)$ because it has a maximal $R$ $\left(R_{\text {best }}=1.24=R_{\text {perf }} \times 0.88\right)$. The $C 1-F 1$ scatter plots for all stations are shown in Fig. S5. There are 16 stations with matching coefficient $R>1$ in the non-end models, excluding DAHU, HERM, SIHU, and FENG. This result suggests that the anomalies of skewness and kurtosis of geoelectric fields catch large earthquakes well, and the geoelectric system would respond to earthquake preparation.

Table 1 inventories the parameters $\left[\operatorname{Rad}_{\text {best }}\right.$, Dep best $\left._{\text {best }}, t 1_{\text {best }}, t 2_{\text {best }}, t 3_{\text {best }}\right]$ for each station, also including the performance $C 1, F 1$, and $R$, as well as the portion of models with $R>1$. Using these best parameters, we generated a GEMSTIP matching diagram for

Table 1 Parameters and null hypothesis test of the best GEMSTIP model

\begin{tabular}{|c|c|c|c|c|c|c|c|c|c|c|c|}
\hline Sta & $\operatorname{Rad}$ & Dep & $N$ & $t 1$ & $t 2$ & $t 3$ & $C 1$ & $F 1$ & $R$ & $P(R>1)$ & $H$ \\
\hline SHRL & 55 & 90 & 3 & 13 & 80 & 80 & 0.93 & 0.55 & 1.08 & 0.0813 & 0 \\
\hline KUOL & 40 & 30 & 1 & 23 & 70 & 50 & 0.99 & 0.98 & 1.39 & 0.6571 & 0 \\
\hline TOCH & 30 & 90 & 2 & 9 & 60 & 20 & 0.95 & 0.54 & 1.09 & 0.0377 & 0 \\
\hline HUAL & 70 & 30 & 3 & 5 & 80 & 10 & 0.97 & 0.29 & 1.01 & 0.0028 & 0 \\
\hline ENAN & 70 & 90 & 1 & 3 & 80 & 80 & 0.63 & 0.89 & 1.09 & 0.0323 & 0 \\
\hline DAHU & 100 & 60 & 3 & 7 & 25 & 5 & 0.96 & 0.20 & 0.98 & 0.0000 & 1 \\
\hline LISH & 50 & 30 & 3 & 5 & 70 & 80 & 0.84 & 0.72 & 1.11 & 0.2501 & 0 \\
\hline SHCH & 50 & 30 & 3 & 3 & 30 & 10 & 0.95 & 0.37 & 1.02 & 0.0020 & 0 \\
\hline HERM & 80 & 30 & 3 & 3 & 70 & 5 & 0.90 & 0.05 & 0.90 & 0.0000 & 0 \\
\hline PULI & 30 & 30 & 3 & 9 & 80 & 80 & 0.95 & 0.80 & 1.24 & 3.8663 & 1 \\
\hline FENL & 30 & 60 & 3 & 3 & 20 & 5 & 0.98 & 0.26 & 1.01 & 0.0020 & 0 \\
\hline SIHU & 80 & 30 & 3 & 7 & 10 & 20 & 0.88 & 0.02 & 0.88 & 0.0000 & 1 \\
\hline DABA & 55 & 30 & 3 & 13 & 30 & 40 & 0.99 & 0.97 & 1.39 & 2.6467 & 1 \\
\hline YULI & 30 & 30 & 2 & 7 & 10 & 15 & 0.97 & 0.44 & 1.07 & 0.1403 & 1 \\
\hline $\mathrm{CHCH}$ & 90 & 30 & 1 & 9 & 15 & 50 & 0.96 & 0.63 & 1.15 & 0.0214 & 0 \\
\hline LIOQ & 100 & 30 & 2 & 11 & 60 & 10 & 0.94 & 0.44 & 1.04 & 0.0102 & 0 \\
\hline RUEY & 90 & 60 & 1 & 11 & 80 & 80 & 0.70 & 0.84 & 1.09 & 0.1512 & 0 \\
\hline $\mathrm{KAOH}$ & 90 & 30 & 1 & 17 & 60 & 60 & 0.99 & 0.96 & 1.38 & 1.8863 & 1 \\
\hline WANL & 80 & 30 & 3 & 5 & 50 & 30 & 0.95 & 0.50 & 1.07 & 0.0226 & 1 \\
\hline FENG & 90 & 30 & 1 & 3 & 5 & 5 & 0.92 & 0.07 & 0.92 & 0.0000 & 1 \\
\hline
\end{tabular}

The 1st column (Sta) is the station code, the 2nd ( $\mathrm{Rad})$ is the detection radius of the station, the 3rd (Dep) is the detection depth of the station, the 4th $(N)$ is the threshold of anomalous index numbers, the 5 th $(t 1)$ is the anomalous time, the 6th $(t 2)$ is the calculation period, the 7 th $(t 3)$ is one TIP length, the 8 th $(C 1)$ is the probability of TN, the 9 th $(F 1)$ is the probability of TP, the 10th $(R)$ is the performance of the model, the 11 th $(P)$ is the ratio of models with $R>1$ and without end parameters to all models, and the 12 th $(H)$ is the null hypothesis testing; The result $H=1$ means rejection of the null hypothesis at $5 \%$ significance level, with $H=0$ otherwise 
catching large earthquakes in the training period (Fig. 5). We observed that the $\{2013 / 03 /$ 27, 121.05E, 23.90N, $\left.M_{\mathrm{L}} 6.24\right\}$ earthquake could be detected by PULI and DABA, the $\left\{2013 / 06 / 02,120.97 \mathrm{E}, 23.86 \mathrm{~N}, M_{\mathrm{L}} 6.48\right\}$ earthquake by PULI, and the $\{2013 / 10 / 31$, $\left.121.35 \mathrm{E}, 23.57 \mathrm{~N}, M_{\mathrm{L}} 6.42\right\}$ earthquake by LIOQ and RUEY. We found that 35 earthquakes with $M_{\mathrm{L}} \in[5,6$ ) (of 50 in this study) were related to anomalies of skewness and kurtosis of the geoelectric fields. Note that there are many oversea earthquakes, which might be beyond the detection sensitivity of the GEMS stations. Finally, we conducted null hypothesis testing to show that the real parameters for all stations were not selected by random cases. Figures S6-S25 show comparisons of the performance and parameters between the real and random cases for all stations. We found that the pair $(C 1, F 1)$ of the real case for each station was more or less enclosed by those of the random cases. This result means that the GEMSTIP model is not biased, and can fit not only the real data but also the noisy data. For the sake of visualization of comparisons of the model parameters, we plotted 3D scatter plots of the triple (Rad, Dep, tmCG) and the triple $(N \times t 1, t 2, t 3)$. Note that the parameter tmCG is the coarse-graining time; in this paper, tmCG $=t 3$. The CGEQ indexes are calculated on the parameters (Rad, Dep, tmCG), while the TIP indexes are based on the parameters $(N, t 1, t 2, t 3)$. We then quantitatively determined whether the parameters of the real case for each station were different from those of the random cases. We compile the null hypothesis testing $H$ for each station in the 12th column of Table 1. We found that the real parameters for DAHU, PULI, SIHU, DABA, YULI, KAOH, WANL, and FENG were distinguished from the random cases. This test suggests that the clustered skewness and kurtosis anomalies in some regions are relevant to earthquakes with high confidence.

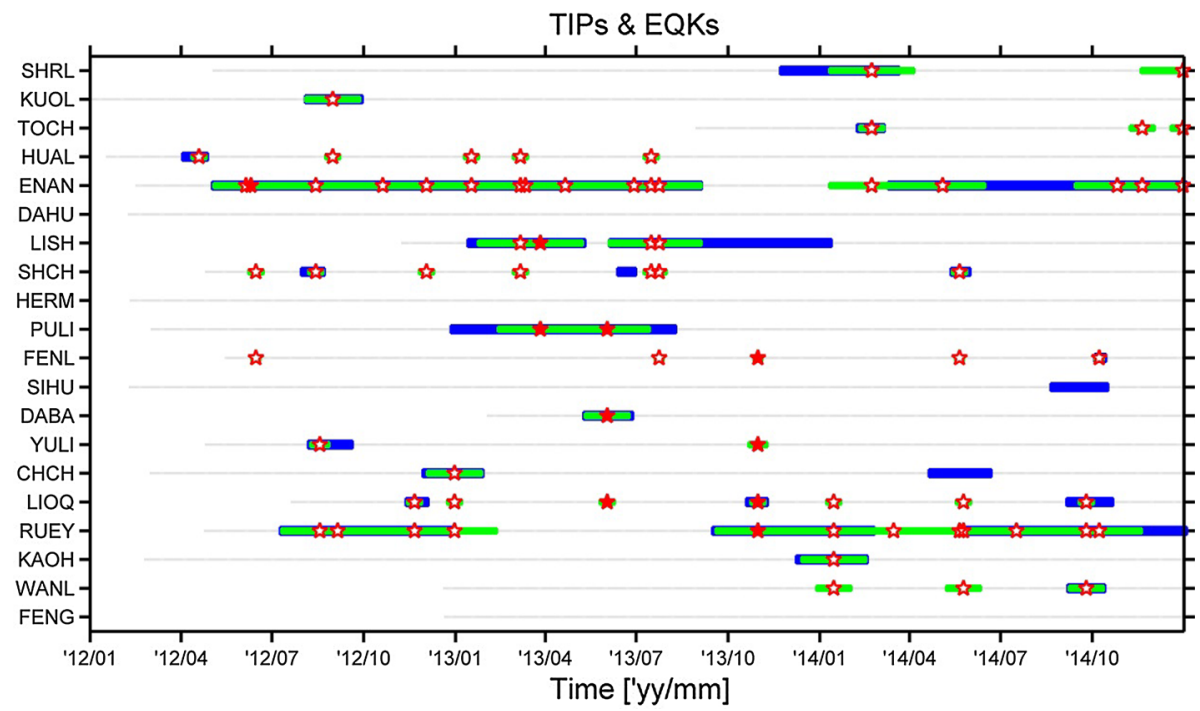

Fig. 5 GEMSTIP matching diagram. Blue horizontal lines are TIPs. Red open stars are earthquakes with $M_{\mathrm{L}} \in[5,6)$, and solid red stars are earthquakes with $M_{\mathrm{L}} \geq 6$. Green horizontals are the time expansions of earthquake occurrence plus and minus $t 3 / 2$. Gray lines are auxiliary; their front ends show the starting times of the stations 


\section{Discussion}

Because the statistical indexes of the geoelectric fields varied immensely, the geoelectric system is considered to be nonlinear and nonstationary. This may be explained by the fact that numerous physical processes transform the lithosphere into a large nonlinear system that is unstable and chaotic (Keilis-Borok 1990). Various phenomena are thus observed and measured in the geoelectric system. The geoelectric system reveals the scaling relationship during the earthquake preparation process (Varotsos et al. 2002; Eftaxias et al. 2003). Secondly, in this work, anomalies of statistical indexes of the geoelectric fields appear before large earthquakes with $M_{\mathrm{L}} \geq 5$. Thirdly, we observed time lags between the climaxes of the clustered anomalies and the earthquake occurrences.

We considered that critical transitions would explain these phenomena of the geoelectric system and earthquakes. Firstly, the studies of Varotsos et al. (2002) and Eftaxias et al. (2003) support the criticality in earthquake ruptures of Sornette and Sornette (1990). They suggested that rupture is a critical point in earthquakes, and proposed a critical theory between the geoelectric system and earthquakes in terms of piezoelectricity. Secondly, rethinking the result that statistical anomalies of geoelectric fields precede earthquakes, we considered that the mechanism of critical transition of Scheffer et al. (2009) may explain those anomalies of skewness and kurtosis well. They proposed early warning signals for critical transitions, and explained why generic symptoms may occur as the system approaches its critical point before catastrophic events. In the theory of critical transition, the state of a system in a double-well potential is permanently subjected to natural perturbations. If one well becomes flatter, the perturbed state in that well will return to its equilibrium gradually and slowly. Meanwhile, the state would shift from one well to the other due to perturbations with higher probability. Measuring the approach to a critical tipping point could be achieved by straightforwardly measuring the recovery rates (resilience) of the state back to its initial equilibrium following perturbations. Note that the state of a system is always driven by perturbations. However, the recovery rates are estimated in theoretical models rather than in reality. Alternatively, there are many types of precursory indicators in complex dynamical systems before critical transitions, including autocorrelation, variance, skewness, kurtosis, etc. (Scheffer et al. 2009). These indexes can be measured in practice. The nonlinear geoelectric system might exhibit the varied dynamics of the double-well potential through different distributions of geoelectric fields; in this study, we indeed observed anomalous skewness and kurtosis before earthquakes. Early warning signals for critical transitions have also been independently identified by using natural time analysis (Sarlis et al. 2013; Varotsos et al. 2008, 2011b). Alternative measures to distinguish precursory geoelectric field anomalies from nonprecursory ones have been proposed on the basis of natural time analysis (Varotsos et al. 2003a, b). In reality, there are many natural systems that may exhibit critical phenomena, such as medicine, global finance, ecosystems, the Earth system, etc.; see, e.g., Natural Time Analysis: The New View of Time (Varotsos et al. 2011a). Note that, prior to critical transitions, skewness may increase or decrease based on whether the transition is towards an alternative state that is larger or smaller than the present state (Dakos et al. 2012). The skewness and kurtosis of geoelectric fields might constitute the signature of an underlying precursory critical mechanism, and the geoelectric fields can thus be used to monitor the crustal system. In alignment with the aims of the present contribution, the results in this paper support the theory of critical transition to explain the connection between the geoelectric system and earthquakes. This theory is consistent with a model (Sarlis et al. 2010; 
Varotsos et al. 1993, 2009; for a brief description of this model see also Varotsos 2007) proposed to explain the generation of precursory transient changes of the geoelectric field observed in Greece, termed SESs, which suggests that SESs are emitted when the gradually increasing stress in the focal area reaches a critical value. Thirdly, the time lags between the anomalous skewness and kurtosis clusters and large earthquakes imply that the catastrophic event does not need to occur suddenly during the critical transition; that is, large earthquakes occur after the critical transitions of the crustal system. The time lag suggests that a large earthquake might be a first-order phase transition process. A firstorder phase transition allows latent heat to exist; that is, the crustal system after the critical transition will accumulate more energy to release a large earthquake.

Additionally, the best detection range $\left(\operatorname{Rad}_{\text {best }}\right.$, Dep best $)$ varied for each station. This variation implies that local geology affects the seismoelectric behavior, in agreement with the experiments of Fukui et al. (2005). The mean of $\operatorname{Rad}_{\text {best }}$ and Depbest for all stations was 65.5 and $43.5 \mathrm{~km}$, respectively. These detection ranges might correspond to earthquakes with $M_{\mathrm{L}} \geq 5$. Besides, the predictive period $t 3$ could be considered as the earthquake preparation period. Lin (2012) found possible slow slips from approximately 6 days before the $1999 M_{\mathrm{w}} 7.6 \mathrm{Chi}-\mathrm{Chi}$ earthquake. In this work, the value of $t 3_{\text {best }}$ ranged from 5 to 80 days, depending on the site. On average, the parameter $t 3_{\text {best }}$ was 36.75 days. This comparison suggests that anomalies in the crust can be detected electromagnetically earlier than mechanically. Moreover, the null hypothesis testing $H$ for all stations did not reject $H^{0}$. This result indicates that the nucleation dynamics is spatially disordered during the earthquake preparation process, hence the anomalies for some sites were related to major earthquakes with high confidence. The fact that most stations had a best model with $R_{\text {best }}>1$ might indicate a relationship between the geoelectric system and earthquakes. The good match between the TIP and CGEQ indexes implies that the geoelectric system is affected during the earthquake preparation process. We infer that the earthquake preparation process affects the parameters of the lithospheric system, and changes of this system influence the geoelectric field in advance. Precursors of earthquakes exist not only in a seismological sense (seismic quiescence and activation) but also in an electromagnetic sense, as also suggested by Varotsos et al. (2011b). Because the dynamics of systems have generic properties and one may ignore the differences of each system (Scheffer et al. 2009), we considered the geoelectric system instead of the crustal stress system. We cannot directly and definitely know the relationship between the geoelectric system and earthquakes, but we first applied mathematics and statistics to determine their relationship, then the mathematical and statistical indexes aided us in constructing a seismoelectric physical model.

\section{Conclusions}

Anomalies of skewness and kurtosis in geoelectric fields may imply that the system in a seismogenic zone is approaching criticality. It is plausible that the evolution of the Earth's crust towards a critical state takes place in not only a seismological but also electromagnetic sense before an earthquake. This is in fundamental agreement with independent findings (Sarlis et al. 2008; Varotsos et al. 2008, 2011b) showing that an approach to criticality can be identified by natural time analysis of small earthquakes that occur after SES initiation and before the mainshock. Most importantly, the methodology presented herein provides a standard procedure for examining the relationship between anomalies of 
geoelectric fields and earthquakes. This procedure might be extended to other geophysical data in the future to define whether precursory indexes are related to earthquakes. Those precursory indexes, once determined to be effective, could help to develop physical models and understand the earthquake preparation process. Well-accepted theories explaining such anomalous phenomena are still lacking in literature. However, the results in this paper support the theory of critical transition to explain the connection between the geoelectric system and earthquakes, in agreement with independent suggestions by other authors that appeared very recently (Varotsos et al. 2013, 2014). Hence, the critical transition helps us to understand theories of seismoelectromagnetism in the crustal system.

Acknowledgments H.-J.C. and C.-C.C. are grateful for research support: MOST104-2116-M-008-013 from the Ministry of Science and Technology (ROC), MOTC-CWB-104-E-02 from the Central Weather Bureau (ROC), and the Department of Earth Science, National Central University (ROC). H.-J.C. is sponsored by grant 105-2917-I-008-002 from the Ministry of Science and Technology (ROC). We thank Guy Ouillon, Didier Sornette, and Yavor Kamer for comments, and two anonymous reviewers, whose reviews improved this manuscript.

Open Access This article is distributed under the terms of the Creative Commons Attribution 4.0 International License (http://creativecommons.org/licenses/by/4.0/), which permits unrestricted use, distribution, and reproduction in any medium, provided you give appropriate credit to the original author(s) and the source, provide a link to the Creative Commons license, and indicate if changes were made.

\section{References}

Abe S, Suzuki N (2009) Determination of the scale of coarse graining in earthquake networks. EPL Europhys Lett 87:48008. doi:10.1209/0295-5075/87/48008

Astuti W, Sediono W, Akmeliawati R, Aibinu AM, Salami MJE (2013) Investigation of the characteristics of geoelectric field signals prior to earthquakes using adaptive STFT techniques. Nat Hazards Earth Syst Sci 13:1679-1686. doi:10.5194/nhess-13-1679-2013

Carpinteri A, Cardone F, Lacidogna G (2010) Energy emissions from failure phenomena: mechanical, electromagnetic, nuclear. Exp Mech 50:1235-1243. doi:10.1007/s11340-009-9325-7

Chang C-H, Wu Y-M, Chen D-Y, Shin T-C, Chin T-L, Chang W-Y (2012) An examination of telemetry delay in the central weather bureau seismic network. Terr Atmos Ocean Sci 23:261. doi:10.3319/TAO. 2011.11.29.01(T)

Dakos V, Carpenter SR, Brock WA, Ellison AM, Guttal V, Ives AR, Kéfi S, Livina V, Seekell DA, van Nes EH, Scheffer M (2012) Methods for detecting early warnings of critical transitions in time series illustrated using simulated ecological data. PLoS One 7:e41010. doi:10.1371/journal.pone.0041010

Eftaxias K, Kapiris P, Polygiannakis J, Peratzakis A, Kopanas J, Antonopoulos G, Rigas D (2003) Experience of short term earthquake precursors with VLF-VHF electromagnetic emissions. Nat Hazards Earth Syst Sci 3:217-228. doi:10.5194/nhess-3-217-2003

Frid V, Bahat D, Goldbaum J, Rabinovitch A, Frid V, Bahat D, Goldbaum J, Rabinovitch A (2000) Experimental and theoretical investigations of electromagnetic radiation induced by rock fracture. Isr $\mathrm{J}$ Earth Sci 49:9-19. doi:10.1560/6LMJ-ABWH-MBEL-FRTY

Fukui K, Okubo S, Terashima T (2005) Electromagnetic radiation from rock during uniaxial compression testing: the effects of rock characteristics and test conditions. Rock Mech Rock Eng 38:411-423. doi:10.1007/s00603-005-0046-7

Hadjicontis V, Mavromatou C (1994) Transient electric signals prior to rock failure under uniaxial compression. Geophys Res Lett 21:1687-1690. doi:10.1029/94GL00694

Hsiao N-C, Wu Y-M, Zhao L, Chen D-Y, Huang W-T, Kuo K-H, Shin T-C, Leu P-L (2011) A new prototype system for earthquake early warning in Taiwan. Soil Dyn Earthq Eng 31:201-208. doi:10. 1016/j.soildyn.2010.01.008

Huang Q (2011) Rethinking earthquake-related DC-ULF electromagnetic phenomena: towards a physicsbased approach. Nat Hazards Earth Syst Sci 11:2941-2949. doi:10.5194/nhess-11-2941-2011

Huang QH, Liu T (2006) Earthquakes and tide response of geoelectric potential field at the Niijima station. Chin J Geophys 49:1745-1754 
Keilis-Borok VI (1990) The lithosphere of the Earth as a nonlinear system with implications for earthquake prediction. Rev Geophys 28:19-34. doi:10.1029/RG028i001p00019

Keilis-Borok VI, Kossobokov VG (1990) Times of increased probability of strong earthquakes $(\mathrm{M} \geq 7.5)$ diagnosed by algorithm M8 in Japan and adjacent territories. J Geophys Res Solid Earth 95:12413-12422. doi:10.1029/JB095iB08p12413

Klein W, Rundle JB, Ferguson CD (1997) Scaling and nucleation in models of earthquake faults. Phys Rev Lett 78:3793-3796. doi:10.1103/PhysRevLett.78.3793

Lacidogna G, Carpinteri A, Manuello A, Durin G, Schiavi A, Niccolini G, Agosto A (2011) Acoustic and electromagnetic emissions as precursor phenomena in failure processes. Strain 47:144-152. doi:10. 1111/j.1475-1305.2010.00750.x

Lin C-H (2012) The possible observation of slow slip events prior to the occurrence of the 1999 Chi-Chi earthquake. Terr Atmos Ocean Sci 23:145. doi:10.3319/TAO.2011.09.23.01(T)

Massey FJ (1951) The Kolmogorov-Smirnov test for goodness of fit. J Am Stat Assoc 46:68-78. doi:10. $1080 / 01621459.1951 .10500769$

Mavromatou C, Hadjicontis V, Ninos D, Mastrogiannis D, Hadjicontis E, Eftaxias K (2004) Understanding the fracture phenomena in inhomogeneous rock samples and ionic crystals, by monitoring the electromagnetic emission during their deformation. Phys Chem Earth Parts ABC 29:353-357. doi:10.1016/ j.pce.2003.11.011

Miller LH (1956) Table of percentage points of Kolmogorov statistics. J Am Stat Assoc 51:111-121. doi:10. $1080 / 01621459.1956 .10501314$

Orihara Y, Kamogawa M, Nagao T, Uyeda S (2012) Variations of geoelectric potential differences associated with an anomalous volumetric strain change in the region of expected Tokai Earthquake, Japan. Nat Hazards Earth Syst Sci 12:121-127. doi:10.5194/nhess-12-121-2012

Ouillon G, Sornette D (2005) Magnitude-dependent Omori law: theory and empirical study. J Geophys Res Solid Earth 110:B04306. doi:10.1029/2004JB003311

Ouillon G, Sornette D, Ribeiro E (2009) Multifractal Omori law for earthquake triggering: new tests on the California, Japan and worldwide catalogues. Geophys J Int 178:215-243. doi:10.1111/j.1365-246X. 2009.04079.x

Sarlis NV, Skordas ES, Lazaridou MS, Varotsos PA (2008) Investigation of seismicity after the initiation of a seismic electric signal activity until the main shock. Proc Jpn Acad Ser B Phys Biol Sci 84:331-343. doi:10.2183/pjab/84.331

Sarlis NV, Skordas ES, Varotsos PA (2010) Nonextensivity and natural time: the case of seismicity. Phys Rev E 82:21110. doi:10.1103/PhysRevE.82.021110

Sarlis NV, Skordas ES, Varotsos PA, Nagao T, Kamogawa M, Tanaka H, Uyeda S (2013) Minimum of the order parameter fluctuations of seismicity before major earthquakes in Japan. Proc Natl Acad Sci 110:13734-13738. doi:10.1073/pnas.1312740110

Scheffer M, Bascompte J, Brock WA, Brovkin V, Carpenter SR, Dakos V, Held H, van Nes EH, Rietkerk M, Sugihara G (2009) Early-warning signals for critical transitions. Nature 461:53-59. doi:10.1038/ nature 08227

Shinbrot T, Kim NH, Thyagu NN (2012) Electrostatic precursors to granular slip events. Proc Natl Acad Sci 109:10806-10810. doi:10.1073/pnas.1121596109

Sornette D, Ouillon G (2005) Multifractal scaling of thermally activated rupture processes. Phys Rev Lett 94:38501. doi:10.1103/PhysRevLett.94.038501

Sornette A, Sornette D (1990) Earthquake rupture as a critical point: consequences for telluric precursors. Tectonophysics 179:327-334. doi:10.1016/0040-1951(90)90298-M

Telesca L, Colangelo G, Lapenna V, Macchiato M (2004) Fluctuation dynamics in geoelectrical data: an investigation by using multifractal detrended fluctuation analysis. Phys Lett A 332:398-404. doi:10. 1016/j.physleta.2004.10.011

Telesca L, Lovallo M, Ramirez-Rojas A, Angulo-Brown F (2009) A nonlinear strategy to reveal seismic precursory signatures in earthquake-related self-potential signals. Phys Stat Mech Appl 388:2036-2040. doi:10.1016/j.physa.2009.01.035

Tiampo KF, Rundle JB, McGinnis S, Gross SJ, Klein W (2002) Mean-field threshold systems and phase dynamics: an application to earthquake fault systems. EPL Europhys Lett 60:481. doi:10.1209/epl/ i2002-00289-y

Triantis D, Anastasiadis C, Stavrakas I (2008) The correlation of electrical charge with strain on stressed rock samples. Nat Hazards Earth Syst Sci 8:1243-1248. doi:10.5194/nhess-8-1243-2008

Tsai C-Y, Ouillon G, Sornette D (2012) New empirical tests of the multifractal Omori law for Taiwan. Bull Seismol Soc Am 102:2128-2138. doi:10.1785/0120110237

Tsutsumi A, Tanaka S, Shirai N, Enomoto Y (2003) Electric signals accompanying fracture of granite. Jpn J Appl Phys 42:5208. doi:10.1143/JJAP.42.5208 
Uyeda S, Hayakawa M, Nagao T, Molchanov O, Hattori K, Orihara Y, Gotoh K, Akinaga Y, Tanaka H (2002) Electric and magnetic phenomena observed before the volcano-seismic activity in 2000 in the Izu Island region, Japan. Proc Natl Acad Sci 99:7352-7355. doi:10.1073/pnas.072208499

Varotsos P (2005) The physics of seismic electric signals. TERRAPUB, Tokyo

Varotsos PA (2007) Calculation of point defect parameters in diamond. Phys Rev B 75:172107. doi:10. 1103/PhysRevB.75.172107

Varotsos P, Alexopoulos K (1984a) Physical properties of the variations of the electric field of the earth preceding earthquakes, I. Tectonophysics 110:73-98. doi:10.1016/0040-1951(84)90059-3

Varotsos P, Alexopoulos K (1984b) Physical properties of the variations of the electric field of the earth preceding earthquakes: II. determination of epicenter and magnitude. Tectonophysics 110:99-125. doi:10.1016/0040-1951(84)90060-X

Varotsos P, Lazaridou M (1991) Latest aspects of earthquake prediction in Greece based on seismic electric signals. Tectonophysics 188:321-347. doi:10.1016/0040-1951(91)90462-2

Varotsos P, Alexopoulos K, Nomicos K, Lazaridou M (1986) Earthquake prediction and electric signals. Nature 322:120. doi:10.1038/322120a0

Varotsos P, Alexopoulos K, Lazaridou M (1993) Latest aspects of earthquake prediction in Greece based on seismic electric signals, II. Tectonophysics 224:1-37. doi:10.1016/0040-1951(93)90055-O

Varotsos PA, Sarlis NV, Skordas ES (2002) Long-range correlations in the electric signals that precede rupture. Phys Rev E 66:011902. doi:10.1103/PhysRevE.66.011902

Varotsos PA, Sarlis NV, Skordas ES (2003a) Long-range correlations in the electric signals that precede rupture: further investigations. Phys Rev E 67:21109. doi:10.1103/PhysRevE.67.021109

Varotsos PA, Sarlis NV, Skordas ES (2003b) Attempt to distinguish electric signals of a dichotomous nature. Phys Rev E 68:31106. doi:10.1103/PhysRevE.68.031106

Varotsos PA, Sarlis NV, Skordas ES, Lazaridou MS (2008) Fluctuations, under time reversal, of the natural time and the entropy distinguish similar looking electric signals of different dynamics. J Appl Phys 103:14906. doi:10.1063/1.2827363

Varotsos PA, Sarlis NV, Skordas ES (2009) Detrended fluctuation analysis of the magnetic and electric field variations that precede rupture. Chaos Interdiscip J Nonlin Sci 19:23114. doi:10.1063/1.3130931

Varotsos PA, Sarlis NV, Skordas ES (2011a) Identifying long-range correlated signals upon significant periodic data loss. Tectonophysics 503:189-194. doi:10.1016/j.tecto.2011.02.011

Varotsos P, Sarlis NV, Skordas ES (2011b) Natural time analysis: the new view of time: precursory seismic electric signals, earthquakes and other complex time series. Springer, Berlin

Varotsos P, Sarlis NV, Skordas ES, Uyeda S, Kamogawa M (2011c) Natural time analysis of critical phenomena. Proc Natl Acad Sci 108:11361-11364. doi:10.1073/pnas.1108138108

Varotsos PA, Sarlis NV, Skordas ES, Lazaridou MS (2013) Seismic electric signals: an additional fact showing their physical interconnection with seismicity. Tectonophysics 589:116-125. doi:10.1016/j. tecto.2012.12.020

Varotsos PA, Sarlis NV, Skordas ES (2014) Study of the temporal correlations in the magnitude time series before major earthquakes in Japan. J Geophys Res Space Phys 119:9192-9206. doi:10.1002/ 2014JA020580

Zhao Y, Qian F (1994) Geoelectric precursors to strong earthquakes in China. Tectonophysics 233:99-113. doi:10.1016/0040-1951(94)90223-2 